\title{
A High-Efficiency Photonic Nanowire Single-Photon Source Featuring An Inverted Conical Taper
}

Gregersen, Niels; Nielsen, Torben Roland; Mørk, Jesper; Claudon, Julien; Gérard, Jean-Michel

Published in:

2011 11th International Conference on Numerical Simulation of Optoelectronic Devices (NUSOD)

Link to article, DOI:

10.1109/NUSOD.2011.6041136

Publication date:

2011

Link back to DTU Orbit

Citation (APA):

Gregersen, N., Nielsen, T. R., Mørk, J., Claudon, J., \& Gérard, J-M. (2011). A High-Efficiency Photonic Nanowire Single-Photon Source Featuring An Inverted Conical Taper. In 2011 11th International Conference on Numerical Simulation of Optoelectronic Devices (NUSOD) (pp. 5-6) https://doi.org/10.1109/NUSOD.2011.6041136

\section{General rights}

Copyright and moral rights for the publications made accessible in the public portal are retained by the authors and/or other copyright owners and it is a condition of accessing publications that users recognise and abide by the legal requirements associated with these rights.

- Users may download and print one copy of any publication from the public portal for the purpose of private study or research.

- You may not further distribute the material or use it for any profit-making activity or commercial gain

- You may freely distribute the URL identifying the publication in the public portal 


\section{A High-Efficiency Photonic Nanowire Single-Photon Source Featuring An Inverted Conical Taper}

\author{
Niels Gregersen, Torben R. Nielsen and Jesper Mørk \\ DTU Fotonik, Department of Photonics Engineering \\ Technical University of Denmark \\ Kongens Lyngby, Denmark \\ ngre@fotonik.dtu.dk
}

\begin{abstract}
A photonic nanowire single-photon source design incorporating an inverted conical tapering is proposed. The inverted taper allows for easy electrical contacting and a high photon extraction efficiency of $89 \%$. Unlike cavity-based approaches, the photonic nanowire features broadband spontaneous emission control and an improved tolerance towards fabrication imperfections.
\end{abstract}

Keywords-photonic nanowire; single-photon source; inverted conical tapering; electrical pumping.

\section{INTRODUCTION}

The solid-state single-photon source (SPS) is a key component in the field of quantum information processing [1]. Here, a photon extraction efficiency $\varepsilon$ close to $100 \%$ is desired, where $\varepsilon$ is defined as the number of detected photons in the collection optics per trigger. Assuming a perfect radiative yield, the efficiency is defined as $\varepsilon=\beta \gamma$ [2], where $\beta$ is the fraction of spontaneous emission coupled from the photon emitter to the optical mode of interest and $\gamma$ is the power collected by the collection optics relative to the power of the optical mode. In the microcavity SPS, the Purcell effect is employed ensure a high $\beta$, however this approach is highly sensitive to fabrication imperfections, limiting $\varepsilon$ to $44 \%$ [3].

Recently, we demonstrated an optically pumped SPS based on a quantum dot (QD) in a photonic nanowire with a regular conical tapering featuring a record-high efficiency of $72 \%$ [4]. Unlike cavity-based SPS designs, a geometrical effect ensures a $\beta>90 \%$ over a broad $70 \mathrm{~nm}$ wavelength range. This tolerance relaxes fabrication constraints and allows for broadband emission control. However, the regular taper is defined using a serial ion beam etching process and is incompatible with direct electrical contacting. To address these issues, we propose in this work a photonic nanowire design incorporating an inverted conical tapering allowing for both parallel processing and easy electrical contacting [5].

\section{NANOWIRE SPS DESIGN}

We study the GaAs nanowire with an embedded QD emitting light at $950 \mathrm{~nm}$ sketched in Fig. 1. The metal contact sections are electrically isolated by air and the GaAs nanowire is doped to create a $p-i-n$ diode.

\author{
Julien Claudon and Jean-Michel Gérard \\ CEA-CNRS-UJF group "Nanophysique et \\ Semiconducteurs" \\ CEA, INAC, SP2M \\ Grenoble, France
}

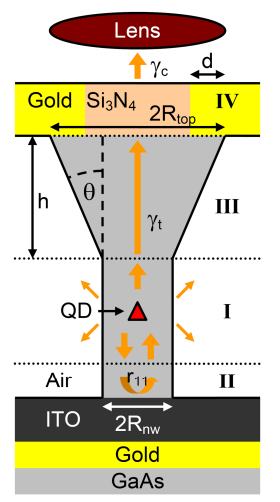

Figure 1. Photonic nanowire design with an inverted conical taper.

The SPS consists of four separate elements. Element I is the nanowire incorporating a QD emitting photons predominantly into the $\mathrm{HE}_{11}$ mode. Half of these propagate downwards, so we include the metal mirror in element II to reflect this light back towards the top. In the central element III, the $\mathrm{HE}_{11}$ mode profile is adiabatically expanded with the purpose of obtaining a low output beam divergence. Finally, element IV features an annular gold contact and a central anti-reflection coating.

Following [6] we employ an elements-splitting approach to compute the total efficiency $\varepsilon$. Here $\varepsilon$ is given by [6]

$$
\varepsilon=\beta \gamma \frac{\left(1+\left|r_{11}\right|\right)^{2}}{2\left(1+\beta\left|r_{11}\right|\right)},
$$

where $r_{11}$ is the modal reflectivity of the metal mirror and $\gamma$ $=\gamma_{c} \gamma_{t}$ is the transmission through the inverted taper and the top contact sections. The fraction on the RHS of (1) is a correction taking into account the reflectivity of the metal mirror.

Using the elements-splitting approach we can study and optimize the four elements individually and subsequently evaluate the total efficiency $\varepsilon$ using (1). The simulations are performed using the eigenmode expansion technique [7] and the tapering section is modeled using a staircase approximation. 


\section{INVERTED CONICAL TAPER}

The novel feature of our electrically pumped nanowire SPS design is the inverted taper of element III. Whereas a conical taper expanding the $\mathrm{HE}_{11}$ profile into air is employed in the optically pumped nanowire design $[4,6]$, our new SPS design features an inverted tapering which instead expands the mode inside the nanowire [5]. The taper is illustrated in Fig. 2(a). (a)

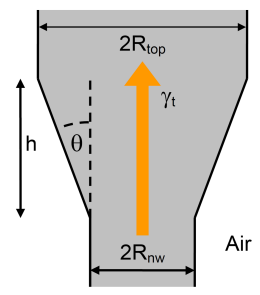

(b)

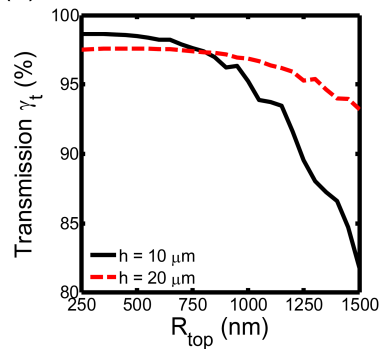

Figure 2. The inverted taper element III (a) and the transmission $\gamma_{t}$ for two different taper heights $h$ as function of $R_{\text {top }}$ (b).

The modal transmission through the taper element is shown in Fig. 2(b) as function of the top nanowire radius $R_{\text {top }}$ for two taper heights $h$. We fix $R_{n w}$ at $120 \mathrm{~nm}$ to ensure a high $\beta$ [5], and we include an absorption loss of $\eta=10 \mathrm{~cm}^{-1}$ due to freecarrier scattering from $n$-doping. For a small $R_{\text {top }}<750 \mathrm{~nm}$, the smaller taper height $h=10 \mu \mathrm{m}$ leads to a smaller absorption loss and a slightly higher transmission than for $h=20 \mu \mathrm{m}$. However, for $R_{\text {top }}>750 \mathrm{~nm}$, the side wall angle $\theta$ becomes large enough to compromise the adiabaticity of the $\mathrm{HE}_{11}$ mode transition. For small values, the side wall angle $\theta$ is given by $\theta$ $\cong\left(R_{\text {top }}-R_{n w}\right) / h$ and the larger taper height $h=20 \mu \mathrm{m}$ results in a smaller side wall angle, a better preservation of adiabaticity and an improved transmission in the regime $R_{t o p}>750 \mathrm{~nm}$.

\section{PREDICTED EFFICIENCY}

From a calculation of the element coefficients $\beta, r_{11}, \gamma_{t}$ and $\gamma_{c}$ we can estimate the total efficiency $\varepsilon$ of the proposed electrically-pumped SPS design. We have the liberty of choosing $R_{\text {top }}, h$ and $d$. In the following we set $d=100 \mathrm{~nm}$, and we study $\varepsilon$ as function of $R_{\text {top }}$ and $h$ in Fig. 3 for a 0.8 NA lens. The figure includes results from the element-splitting approach as well as from exact calculations performed by placing a dipole in the full structure and computing the far field emission profile.

We observe that the efficiency $\varepsilon$ increases with $R_{\text {top }}$ towards a maximum value of $\sim 89 \%$ obtained for $R_{\text {top }} \sim 900 \mathrm{~nm}$. At this radius the collection efficiency $\gamma_{c}$ is no longer improved by increasing $R_{\text {top }}$. A slight deviation for $R_{\text {top }}>750 \mathrm{~nm}$ for the $h=$ $10 \mu \mathrm{m}$ curves computed using the simplified and the exact models is observed. The efficiency obtained by the exact model actually increases beyond $89 \%$ as $R_{\text {top }}$ approaches $1500 \mathrm{~nm}$, whereas $\varepsilon$ for the simplified model clearly decreases, reflecting the drop in transmission observed in Fig. 2(b) for $R_{t o p}$ approaching $1500 \mathrm{~nm}$.

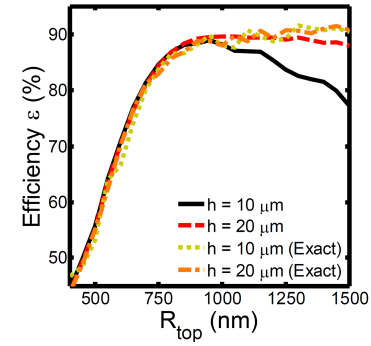

Figure 3. Total SPS efficiency $\varepsilon$ as function of $R_{\text {top }}$ for two different taper heights $h$.

This discrepancy can be understood by considering the breakdown of the adiabatic transition of the $\mathrm{HE}_{11}$ mode as it propagates through the inverted taper. For a fixed taper height $h$, the side wall $\theta$ increases with $R_{\text {top }}$ and for $R_{\text {top }}>1000 \mathrm{~nm}$ there is significant coupling to higher order modes $\mathrm{HE}_{1 m}(m>$ $1)$. In the simplified model this coupling is treated as a loss reducing $\varepsilon$, however even though these higher order modes generally have a more divergent far field emission profile, a significant part of the light from these modes can be picked up by the $0.8 \mathrm{NA}$ lens. The exact computation includes these higher-order contributions, and the redistribution of power to several $\mathrm{HE}_{1 m}$ modes does not decrease $\varepsilon$.

\section{CONCLUSION}

In this work we have demonstrated a practical design for a high-efficiency single-photon source. The central feature of the design is an inverted conical taper allowing simultaneously for easy electrical contacting and a photon extraction efficiency of $89 \%$.

\section{REFERENCES}

[1] A. J. Shields, "Semiconductor quantum light sources," Nat. Photonics, vol. 1, pp. 215-223, April 2007.

[2] W. L. Barnes, G. Björk, J. M. Gérard, P. Jonsson, J. A. E. Wasey, P. T. Worthing and V. Zwiller, "Solid-state single photon sources: light collection strategies," Eur. Phys. J. D, vol. 18, pp. 197-210, February 2002 .

[3] E. Moreau, I. Robert, L. Manin, V. Thierry-Mieg, J. M. Gérard and I. Abram, "A single-mode solid-state source of single photons based on isolated quantum dots in a micropillar" Physica E, vol. 13, pp. 418-422, March 2002.

[4] J. Claudon, J. Bleuse, N. S. Malik, M. Bazin, P. Jaffrennou, N. Gregersen, C. Sauvan, P. Lalanne and J. M. Gérard, "A highly efficient single-photon source based on a quantum dot in a photonic nanowire," Nat. Photonics, vol. 4, pp. 174-177, March 2010.

[5] N. Gregersen, T. R. Nielsen, J. Mørk, J. Claudon and J. M. Gérard, "Designs for high-efficiency electrically pumped photonic nanowire single-photon sources", Opt. Express, vol. 18, pp. 21204-21218, September 2010.

[6] I. Friedler, C. Sauvan, J. P. Hugonin, P. Lalanne, J. Claudon and J. M. Gérard, "Solid-state single photon sources: the nanowire antenna," Opt. Express, vol. 17, pp. 2095-2110, February 2009.

[7] P. Bienstman and R. Baets, "Optical modelling of photonic crystals and VCSELs using eigenmode expansion and perfectly matched layers," Opt. Quantum Electron, vol. 33, pp. 327-341, April 2001. 